\title{
Modelo matemático para estimar la producción de la energía primaria en Ecuador.
}

Mathematical model to estimate the production of primary energy in Ecuador.

\author{
Guido Javier Mazón Fierro. ${ }^{1}$, Pablo Ricardo Calderón Limaico. ${ }^{2}$, Ruffo Neptalí Villa \\ Uvidia. ${ }^{3} \&$ Jenny Margoth Villamarín Padilla. ${ }^{4}$
}

Recibido: 10-03-2019 / Revisado: 15-03-209 /Aceptado: 04-04-2019/ Publicado: 13-05-2019

\begin{abstract}
.
DOI: https://doi.org/10.33262/cienciadigital.v3i2.2.464

In the present paper a series of values of primary energy production in Ecuador was estimated through the multiplicative mathematical model of time series that has as variables the trend, seasonality and noise, it was considered a time lapse of 12 years as of 2015 arriving to obtain estimated values up to the year 2027, as a first step, historical data of the national energy balance of Ecuador 2016 were taken, with these inputs as a starting point the trend was found from a linear extrapolation model, arriving to determine the behavior of the production of primary energy through the trend line: $\mathrm{T}=204.28+1.0956 * \mathrm{t}$ where $\mathrm{T}=$ trend, $\mathrm{t}=$ time series, then the seasonality is analyzed which is another of the variables to be considered in the model, for this it had to adjust or soften the seasonality to reduce the noise and it was achieved with the technique of the third order moving averages, Finally, the multiplicative model with which estimated values were obtained from 2016 to 2027 was applied, it was possible to predict that for the last data of the time series the
\end{abstract}

\footnotetext{
${ }^{1}$ Escuela Superior Politécnica de Chimborazo, Facultad de Administración de Empresas. Riobamba, Ecuador. guido.mazon@espoch.edu.ec

${ }^{2}$ Escuela Superior Politécnica de Chimborazo, Facultad de Administración de Empresas. Riobamba, Ecuador. pablo.calderon@espoch.edu.ec

${ }^{3}$ Escuela Superior Politécnica de Chimborazo, Facultad de Administración de Empresas. Riobamba, Ecuador. ruffo.villa@espoch.edu.ec

${ }^{4}$ Escuela Superior Politécnica de Chimborazo, Facultad de Administración de Empresas. Riobamba, Ecuador. jenny.villamarin@espoch.edu.ec
} 
energy production is $241,832 \mathrm{kBEP}$ (Kilo equivalent barrels of oil), being the highest value of registered production.

Keywords: Model, Projection, Mathematics, Energy, Predict, Production.

\section{Resumen.}

En el presente artículo se estimó una serie de valores de producción de energía primaria en Ecuador mediante el modelo matemático multiplicativo de series temporales que tiene como variables la tendencia, estacionalidad y ruido, se consideró un lapso de tiempo de 12 años a partir del 2015 llegando a obtener valores estimados hasta el año 2027, como primer paso se tomaron datos históricos del balance energético nacional de Ecuador 2016, con estos insumos como punto de partida se encontró la tendencia a partir de un modelo de extrapolación lineal, llegando a determinar el comportamiento de la producción de energía primaria a través de la línea de tendencia: $T=204,28+1.0956 * t$ donde $T=$ tendecia,$\quad t=$ serie de tiempo, a continuación se analiza la estacionalidad que es otro de las variables a considerar en el modelo, para esto se tuvo que ajustar o suavizar la estacionalidad para disminuir el ruido y se lo consiguió con la técnica de las medias móviles de tercer orden, se obtuvo como resultado el índice de estacionalidad corregido, finalmente se aplica el modelo multiplicativo con el cual se consiguió valores estimados desde el año 2016 hasta 2027, se pudo predecir que para el último dato de la serie temporal la producción de energía es $241.832 \mathrm{kBEP}$ (Kilo barriles equivalentes de petróleo), siendo el valor más alto de producción registrado.

Palabras claves: Modelo, Proyección, Matemática, Energía, Predecir, Producción.

\section{Introducción.}

La energía es un pilar en el desarrollo de los países. (Garrido, 2009) menciona que "Uno de los principales vectores de nuestra evolución ha sido y es, sin lugar a dudas, la energía. Ésta ha hecho posible que el ser humano no solo poblara prácticamente la totalidad de la superficie del planeta, sino que se ha llegado al espacio. La energía es fuente de calor, de luz, hace posible que nos desplacemos, que cocinemos nuestros alimentos, que fabriquemos máquinas entre otras cosas".

El modelo actual de desarrollo se sustenta, en gran medida, sobre el consumo de combustibles fósiles: petróleo, gas natural y carbón, que según el Programa de las Naciones Unidas para el desarrollo representan, conjuntamente, más del $80 \%$ del suministro de energía primaria a nivel mundial, los combustibles fósiles tienen su origen en la fotosíntesis de las plantas, las cuales extraen del sol la energía necesaria para desarrollarse gracias a ella absorben dióxido de carbono de la atmósfera y se quedan con la parte que les interesa, esto es, el carbono, devolviendo el oxígeno al medio ambiente. En definitiva, se podría 
considerar a las plantas como unos captadores y acumuladores de energía solar, transformando ésta en carbono. Además, la acumulación de grandes cantidades de materia orgánica en estructuras sedimentarias, sometida a altas presiones y temperaturas, tras un largo periodo de transformación del orden de millones de años, da lugar a los combustibles fósiles. Normalmente, se considera que el carbón procede de depósitos de materia orgánica vegetal terrestre, mientras que el petróleo y el gas natural proceden de depósitos de materia orgánica de origen marino, como algas, plancton.(Garrido, 2009).

Nadie puede desconocer que la energía es el motor que mueve el mundo, sin embargo, involucra problemas serios para la humanidad y el planeta, (Cano, 2014) señala que existe una estrecha relación entre desarrollo, energía y entorno ambiental, la necesidad de utilizar combustibles fósiles va acompañado por el deterioro medioambiental asociado al cambio climático, es un fenómeno que desafía a todos los modelos estadísticos de predicción energética, esto genera un reto para propiciar el incremento en la actividad científica dirigida a identificar y desarrollar fuentes primarias de energía sustentables, sostenibles y con balance energético positivo.

Es por esto que se pretende realizar un aporte en el sector energético mediante un estudio de un modelo matemático para estimar la producción de la energía primaria del Ecuador debido a la importancia de la energía en el desarrollo de cada país y sus implicaciones en el ambiente, como datos para el estudio, se cuenta con el balance energético de Ecuador del año 2016, el cual describe en detalle la matriz energética de manera global y desglosa datos históricos estadísticos anuales de la oferta de energía primaria y la demanda de energía, en kilo barriles equivalentes de petróleo (Kbep), es por ello que el objetivo de este trabajo es determinar la producción de energía primaria en Ecuador al año 2027 para contribuir a las acertadas toma de decisiones.

\section{Metodología}

\section{Marco Teórico Referencial}

Para estudiar un sistema, un modelo matemático comienza con la identificación de los aspectos principales o determinantes del sistema y los caracteriza a través de las expresiones matemáticas. La idea en la construcción es encontrar un equilibrio entre la simplicidad y una reproducción del comportamiento que permita comprender, analizar y predecir, al cambiar el valor de la o las variables que lo describen, la respuesta del sistema en su conjunto.Un modelo matemático es la representación simplificada de la realidad, mediante el uso de funciones que describen su comportamiento, o de ecuaciones que representan sus relaciones.(Bocco, 2010).

En el área energética se puede evidenciar varios casos de estudio sobre las proyecciones a mediano y largo plazo sobre la producción y la demanda de recursos energéticos, se va a citar el estudio realizado en Argentina sobre el Informe de actualización de prospectiva 
energética del año 2016 desarrollada por el Área de prospectiva Energía Eléctrica de ese país, en el cual contiene análisis y reflexiones sobre la problemática de la prospectiva energética en argentina y algunas ideas de orientación para el trabajo a desarrollar por los grupos de investigación para hacer pronósticos en series de tiempo, se ha trabajado en colaboración con la Universidad Tecnológica Nacional - Facultad Regional General Pacheco y con destacados especialistas, con el propósito de asociar el trabajo de investigación con las necesidades genuinas de la sociedad, entendiendo el concepto de planeamiento energético como un conjunto de actividades específicas orientadas no a predecir el futuro sino a emitir hipótesis razonables fundadas en el análisis y el conocimiento, acciones capaces de trasformar y modificar el sector energético.(Canabal \& Marcel, 2009)

Otro caso de estudio es el desarrollado en México el cual lleva por título Consumo de electricidad y crecimiento económico en México análisis de series de tiempo y prospectiva en donde se considera el desarrollo de algoritmos de diagnóstico y predicción de activos en el sector energético, con la finalidad de conocer su estado real y, analizar la operatividad y vida útil de los mismos en el futuro.(Recalde, 2010)

Por serie de tiempo nos referimos a datos estadísticos que se recopilan, observan o registran en intervalos de tiempo regulares diario, semanal, semestral, anual, entre otros. Las componentes de la serie de tiempo son tres tipos básicos de variación, los cuales sobrepuestos o actuando en conjunto, contribuyen a los cambios observados en un período de tiempo y dan a la serie su aspecto característico. Estas tres componentes son: Tendencia, estacionalidad, y variación irregular.(Peña, 2010)

En ambos casos se realizan análisis de pronóstico a futuro, Ahora bien, qué se entiende por pronóstico y cuál es su diferenciación con proyección y perspectivas, términos que se confunde frecuentemente. Por pronóstico se entiende una afirmación sobre el futuro, la cual informa que, bajo determinadas condiciones, en un momento y lugar determinados sucederá un acontecimiento o acontecimientos con una probabilidad muy próxima a la seguridad. Por lo que todo pronóstico significa una afirmación basada en una teoría perfecta, según la moderna lógica científica. En la práctica muy raras veces puede establecerse un pronóstico en el sentido exacto del término, ya que las teorías ni son perfectas ni lo suficientemente amplias para que puedan abarcar todos los factores endógenos y exógenos. Por consiguiente, en la práctica, no se trata de definir con el término pronóstico ninguna predicción cuya certeza esté vinculada al máximo grado de probabilidad o cuyas hipótesis no tengan contenido informativo o sean meras tautologías. En la práctica, sin embargo, sería más adecuado en la mayoría de los casos hablar de proyección y no de pronóstico. Por proyección se entiende una afirmación sobre el futuro desarrollo condicionando a determinadas premisas que sólo posee una probabilidad limitada. Por consiguiente, las predicciones son, hoy por hoy, proyecciones y no 
pronósticos, aunque si bien se utilizan ambos términos. Se puede distinguir las proyecciones de puntos y las de intervalos. El término perspectivas se utiliza más en casos de previsiones a largo plazo, basándose en magnitudes futuras $y$ no en meras extrapolaciones tendenciales. (Martínez et al., 2012)

\section{Marco Metodológico}

En el presente trabajo se va a utilizar un modelo matemático para obtener una proyección o estimación a futuro de la energía primaria en Ecuador, en base a lo mencionado se considera como punto de partida el balance energético nacional del Ecuador año 2016, el cual cuenta con datos históricos de la producción primaria de energía, los mismos que son la base para poder hacer la predicción en el futuro, en el modelo se han considerado dos parámetros como son la tendencia y la estacionalidad, para obtener una confiable proyección de la variable a analizar se utilizó el modelo de secuencia temporal.

Como primer paso se representan los datos años y producción primaria de energía mediante un gráfico de líneas Gráfico 1, en el cual se describe la relación que ha sucedido en pasados años con los datos, después de este paso se utiliza un modelo de extrapolación lineal para obtener una línea de tendencia de los datos esto para proyectar patrones establecidos del pasado hacia el futuro.

Una vez obtenida la línea de tendencia, esta servirá para proyectar o extrapolar la variable producción de energía primaria, se necesita utilizar una serie de tiempo en años para predecir los valores con un modelo de extrapolación lineal.

A continuación, para que el modelo brinde un nivel de confianza y se pueda aceptar su predicción se va ajustar el modelo a partir de dos componentes tendencia y estacionalidad se analizan los datos históricos a ver si responden a un modelo aditivo o un modelo multiplicativo para esto se calcula la serie de diferencias y de cocientes consecutivos con estos dos conjuntos de datos se procede a encontrar su desviación estándar y la media, la relación entre estas dos medidas estadísticas permiten encontrar el coeficiente de variación (CV), siendo el discrimínate para determinar a qué modelo obedecen los datos de producción de energía primaria, como el resultado de CV es menor en la serie de cocientes consecutivos se establece que el modelo multiplicativo es el idóneo para ser utilizado.

$$
\text { Modelo multiplicativo: } Y=T * E * R \quad \text { Ecuación } 1
$$

Donde:

$Y=$ Datos originales

$T=$ Tendencia

$E=$ Estacionalidad

$R=$ Ruido 
De la ecuación 1 se despeja el producto estacionalidad y ruido obteniendo:

$$
E * R=\frac{Y}{T} \quad \text { Ecuación } 2
$$

Como se puede apreciar la estacionalidad se encuentra interferida por el ruido para aislar y disipar la variable ruido y obtener un modelo óptimo, se utilizó las medias móviles de orden tres, para hallar un índice de estacionalidad con el menor ruido posible se dividió la estacionalidad con ruido por las medias móviles y se multiplico por cien.

$$
\text { Iest }=\frac{E * R}{M m 3} * 100 \quad \text { Ecuación } 3
$$

Donde:

Iest $=$ Indice de Estacionalidad

$E * R=$ Estacionalidad con ruido

Mm3 = Medias moviles de grado 3

Se prosigue con la sumatoria de Iest este valor tiene que ser igual a 1200 puesto que tenemos 12 datos originales y estamos utilizando un índice de estacionalidad, como el resultado excede al esperado se lo corrigió mediante la siguiente razón:

$$
\text { IEC }=\frac{\text { Iest }}{\sum_{i=1}^{n=12} \text { Iest }} * 1200 \quad \text { Ecuación } 4
$$

Finalmente se hace uso del modelo matemático multiplicativo para la estimación o predicción de la energía primaria a partir de las componentes de tendencia y estacionalidad:

$$
Y_{E}=\frac{T * I E C}{100} \quad \text { Ecuación } 5
$$

Donde:

$Y_{E}=$ Valores estimados

$T=$ Tendencia

IEC $=$ Indice de Estacionalidad Corregido

Estos valores estimados se los calcula para 12 años que es considerado como plazo de tiempo medio, como se poseen datos originales del año 2004 al 2015 se extrapola el mismo número de datos originales empleando la ecuación 5 , mediante este procedimiento que se obtienen valores futuros de la producción de energía primaria en Ecuador hasta el año 2027. 


\section{Análisis de los Resultados.}

Tabla 1. Producción de energía primaria por años en Ecuador.

\begin{tabular}{cc}
\hline Año & $\begin{array}{c}\text { Producción Energía Primaria en } \\
\text { kBEP(Kilo barriles equivalentes de } \\
\text { petróleo) }\end{array}$ \\
\hline 2004 & 207.509 \\
\hline 2005 & 214.974 \\
\hline 2006 & 216.811 \\
\hline 2007 & 208.488 \\
\hline 2008 & 207.577 \\
\hline 2009 & 198.677 \\
\hline 2010 & 197.970 \\
\hline 2011 & 205.250 \\
\hline 2012 & 208.894 \\
\hline 2013 & 216.074 \\
\hline 2014 & 229.587 \\
\hline 2015 & 225.021 \\
\hline
\end{tabular}

Fuente: Balance energético nacional del Ecuador año 2016.

Gráfico 1. Producción de energía primaria por años en Ecuador y su línea de tendencia.

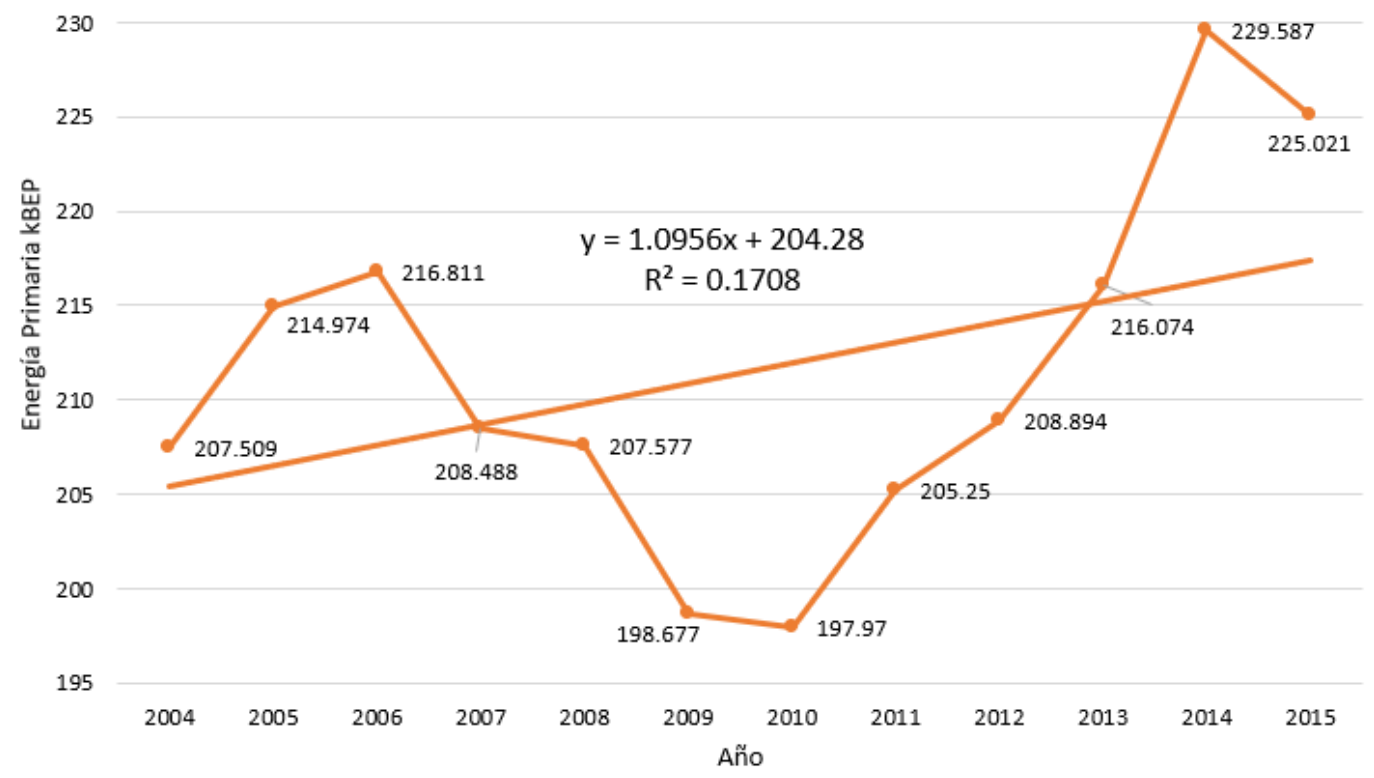

Fuente: Elaboración propia tomado datos del balance energético nacional del Ecuador año 2016.

Con los datos de la Tabla 1 se aplica un modelo de extrapolación lineal para obtener una línea de tendencia: $T=204,28+1.0956 * t \quad$ Ecuación 6

Donde:

$T=$ Tendencia 
$t=$ Serie de tiempo

Tabla 2. Tendencia de la producción de energía primaria por años en Ecuador.

\begin{tabular}{|c|c|c|}
\hline Año & $\begin{array}{c}\text { Tiempo } \\
t\end{array}$ & $\begin{array}{c}\text { Tendencia } \\
T=204,28+1.0956 \\
* t\end{array}$ \\
\hline 2004 & 01 & 205.3756 \\
\hline 2005 & 02 & 206.4712 \\
\hline 2006 & 03 & 207.5668 \\
\hline 2007 & 04 & 208.6624 \\
\hline 2008 & 05 & 209.7580 \\
\hline 2009 & 06 & 210.8536 \\
\hline 2010 & 07 & 211.9492 \\
\hline 2011 & 08 & 213.0448 \\
\hline 2012 & 09 & 214.1404 \\
\hline 2013 & 10 & 215.2360 \\
\hline 2014 & 11 & 216.3316 \\
\hline 2015 & 12 & 217.4272 \\
\hline
\end{tabular}

Fuente: Elaboración propia.

El análisis de datos históricos mediante la serie de diferencias y de cocientes consecutivos nos arroga los siguientes resultados.

Tabla 3. Serie de diferencias y de cocientes consecutivos.

\begin{tabular}{ccrr}
\hline Año & $\begin{array}{c}\text { Producción Energía } \\
\text { Primaria kBEP }\end{array}$ & $\begin{array}{c}\text { Serie } \\
\text { diferencias } \\
\text { consecutivas }\end{array}$ & $\begin{array}{c}\text { Serie } \\
\text { cocientes } \\
\text { consecutivos }\end{array}$ \\
\hline 2004 & 207.509 & 7.465 & 1.03597 \\
\hline 2005 & 214.974 & 1.837 & 1.00855 \\
\hline 2006 & 216.811 & -8.323 & 0.96161 \\
\hline 2007 & 208.488 & -0.911 & 0.99563 \\
\hline 2008 & 207.577 & -8.900 & 0.95712 \\
\hline 2009 & 198.677 & -0.707 & 0.99644 \\
\hline 2010 & 197.970 & 7.280 & 1.03677 \\
\hline 2011 & 205.250 & 3.644 & 1.01775 \\
\hline 2012 & 208.894 & 7.180 & 1.03437 \\
\hline 2013 & 216.074 & 13.513 & 1.06254 \\
\hline 2014 & 229.587 & -4.566 & 0.98011 \\
\hline 2015 & 225.021 & 1.59200 & 1.00790 \\
\hline \multicolumn{5}{c}{ Media } & 6.75757 & 0.03188 \\
\hline Desviación estándar sd & 4.24471 & 0.03163 \\
\hline Coeficiente de variación CV
\end{tabular}

Fuente: Balance energético nacional del Ecuador año 2016. 
El coeficiente de variación (CV), determina a qué modelo obedecen los datos de producción de energía primaria: CV Serie de cocientes $<$ CV Serie de diferencias

$$
0.03163<4.24471
$$

como el resultado de $\mathrm{CV}$ es menor en la serie de cocientes consecutivos se establece que el modelo multiplicativo es el idóneo para ser utilizado.

Posteriormente en la Tabla 4 se presentan valores de estacionalidad con ruido o fluctuaciones.

Tabla 4. Estacionalidad con ruido.

\begin{tabular}{cccc}
\hline Año & $\begin{array}{c}\text { Producción } \\
\text { Energía } \\
\text { Primaria kBEP } \\
Y\end{array}$ & $\begin{array}{c}\text { Tendencia } \\
T\end{array}$ & $\begin{array}{c}\text { Estacionalidad } \\
\text { con Ruido }\end{array}$ \\
\hline 2004 & 207.509 & 205.3756 & 1.010388 \\
\hline 2005 & 214.974 & 206.4712 & 1.041182 \\
\hline 2006 & 216.811 & 207.5668 & 1.044536 \\
\hline 2007 & 208.488 & 208.6624 & 0.999164 \\
\hline 2008 & 207.577 & 209.7580 & 0.989602 \\
\hline 2009 & 198.677 & 210.8536 & 0.942251 \\
\hline 2010 & 197.970 & 211.9492 & 0.934045 \\
\hline 2011 & 205.250 & 213.0448 & 0.963412 \\
\hline 2012 & 208.894 & 214.1404 & 0.975500 \\
\hline 2013 & 216.074 & 215.2360 & 1.003893 \\
\hline 2014 & 229.587 & 216.3316 & 1.061274 \\
\hline 2015 & 225.021 & 217.4272 & 1.034926 \\
\hline
\end{tabular}

Fuente: Balance energético nacional del Ecuador año 2016.

Para ajustar o modelizar la Estacionalidad y disipar el ruido se calcula el Índice de estacionalidad corregido cuyas soluciones se indican en la siguiente tabla:

Tabla 5. Índice de estacionalidad corregido.

\begin{tabular}{lccrr}
\hline Año & $\begin{array}{c}\text { Estacionalidad } \\
\text { con Ruido } \\
E * R=\frac{Y}{T}\end{array}$ & $\begin{array}{c}\text { Medias } \\
\text { Móviles } \\
\text { de grado } \\
\mathbf{3}\end{array}$ & $\begin{array}{c}\text { Índice de } \\
\text { estacionalidad } \\
\text { Iest }=\frac{E * R}{M m 3} * 100\end{array}$ & $\begin{array}{c}\text { Índice de } \\
\text { estacionalidad } \\
\text { corregido } \\
I E C\end{array}$ \\
\hline 2004 & 1.010388 & & 99.929478 & 99.340571 \\
\hline 2005 & 1.041182 & 1.032035 & 100.886250 & 100.291704 \\
\hline 2006 & 1.044536 & 1.028294 & 101.579520 & 100.980888 \\
\hline 2007 & 0.999164 & 1.011101 & 98.819441 & 98.237075 \\
\hline 2008 & 0.989602 & 0.977006 & 101.289296 & 100.692374 \\
\hline 2009 & 0.942251 & 0.955299 & 98.634110 & 98.052836 \\
\hline 2010 & 0.934045 & 0.946569 & 98.676830 & 98.095304 \\
\hline 2011 & 0.963412 & 0.957652 & 100.601472 & 100.008604 \\
\hline
\end{tabular}


Vol. 3, N².2., p. 118-131, abril - junio, 2019

\begin{tabular}{rrrrr}
\hline 2012 & 0.975500 & 0.980935 & 99.445923 & 98.859865 \\
\hline 2013 & 1.003893 & 1.013556 & 99.046692 & 98.462987 \\
\hline 2014 & 1.061274 & 1.033364 & 102.700821 & 102.095581 \\
\hline 2015 & 1.034926 & & 105.503970 & 104.882211 \\
\hline & & Sumatoria & 1207.1138 & 1200 \\
\hline
\end{tabular}

Fuente: Elaboración propia.

Tabla 6. Estimación de la producción de la energía primaria en Ecuador.

\begin{tabular}{|c|c|c|c|c|}
\hline Año & $\begin{array}{c}\text { Tiempo } \\
t\end{array}$ & $\begin{array}{c}\text { Tendencia } \\
T\end{array}$ & $\begin{array}{c}\text { Índice de } \\
\text { estacionalidad } \\
\text { corregido } \\
\text { IEC }\end{array}$ & $\begin{array}{c}\text { Valores } \\
\text { Estimados } \\
Y_{E}\end{array}$ \\
\hline 2004 & 1 & 205.3756 & 99.340571 & 204.021 \\
\hline 2005 & 2 & 206.4712 & 100.291704 & 207.073 \\
\hline 2006 & 3 & 207.5668 & 100.980888 & 209.603 \\
\hline 2007 & 4 & 208.6624 & 98.237075 & 204.984 \\
\hline 2008 & 5 & 209.758 & 100.692374 & 211.210 \\
\hline 2009 & 6 & 210.8536 & 98.052836 & 206.748 \\
\hline 2010 & 7 & 211.9492 & 98.095304 & 207.912 \\
\hline 2011 & 8 & 213.0448 & 100.008604 & 213.063 \\
\hline 2012 & 9 & 214.1404 & 98.859865 & 211.699 \\
\hline 2013 & 10 & 215.236 & 98.462987 & 211.928 \\
\hline 2014 & 11 & 216.3316 & 102.095581 & 220.865 \\
\hline 2015 & 12 & 217.4272 & 104.882211 & 228.042 \\
\hline 2016 & 13 & 218.5228 & 99.340571 & 217.082 \\
\hline 2017 & 14 & 219.6184 & 100.291704 & 220.259 \\
\hline 2018 & 15 & 220.714 & 100.980888 & 222.879 \\
\hline 2019 & 16 & 221.8096 & 98.237075 & 217.899 \\
\hline 2020 & 17 & 222.9052 & 100.692374 & 224.449 \\
\hline 2021 & 18 & 224.0008 & 98.052836 & 219.639 \\
\hline 2022 & 19 & 225.0964 & 98.095304 & 220.809 \\
\hline 2023 & 20 & 226.192 & 100.008604 & 226.211 \\
\hline 2024 & 21 & 227.2876 & 98.859865 & 224.696 \\
\hline 2025 & 22 & 228.3832 & 98.462987 & 224.873 \\
\hline 2026 & 23 & 229.4788 & 102.095581 & 234.288 \\
\hline 2027 & 24 & 230.5744 & 104.882211 & 241.832 \\
\hline
\end{tabular}

Fuente: Elaboración propia. 
Gráfico 2. Valores estimados de Producción de energía primaria en Ecuador.

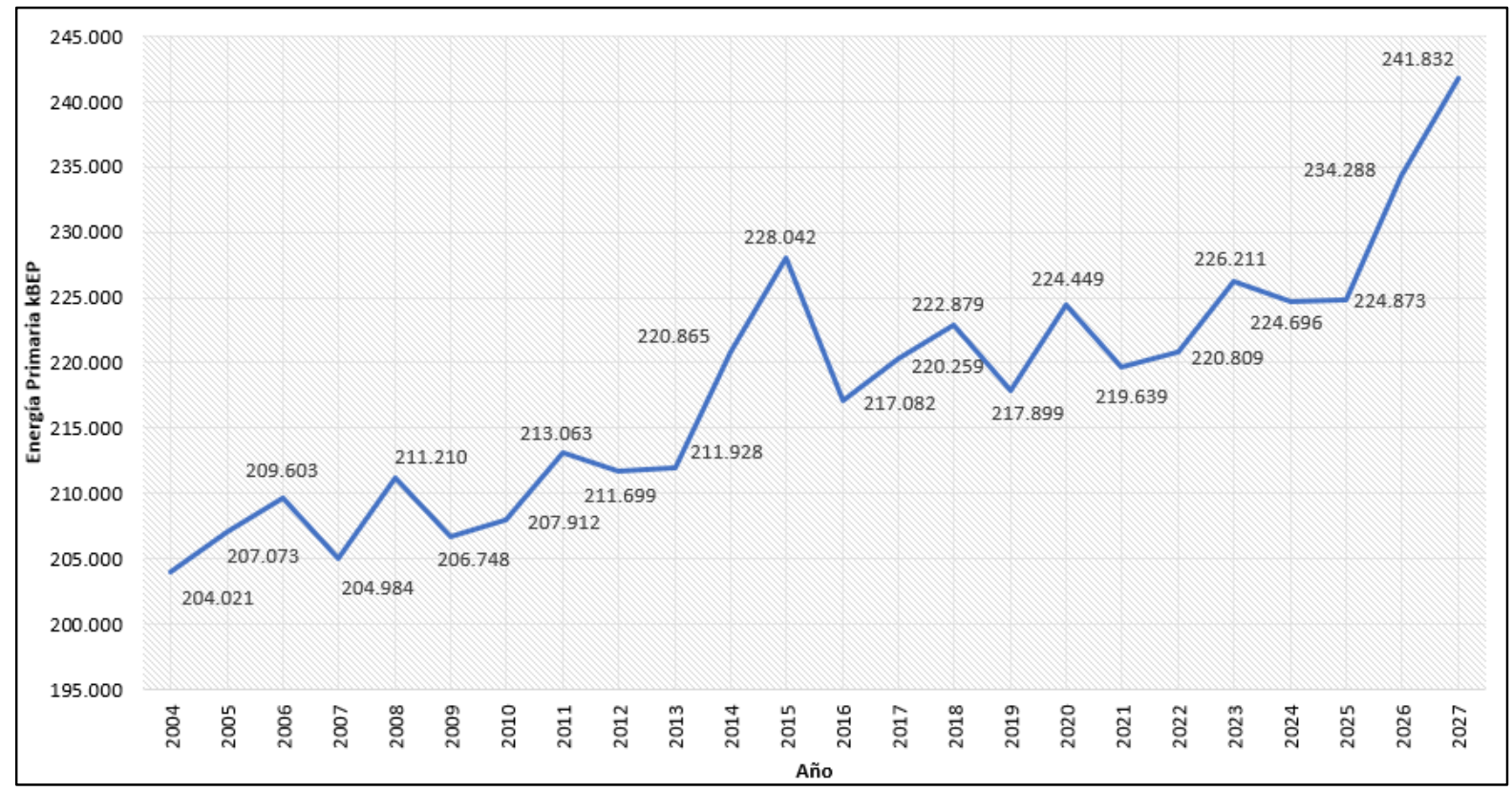

Fuente: Elaboración propia.

\section{Conclusiones.}

- Se ha estimado mediante un modelo matemático multiplicativo una serie de valores de la producción de energía primaria en Ecuador a mediano plazo en 12 años, se puede predecir que para el año 2027 aumentara la producción de energía a 241.832 kBEP (Kilo barriles equivalentes de petróleo), siendo el valor más alto de producción que se tiene que generar para llegar a este requerimiento, esto anuncia que se debe desarrollar otras fuentes de energía posiblemente renovables para cubrir los kBEP que se necesitan a mediano plazo.

- El modelo matemático para la naturaleza de los datos que se analizaron se comprobó que cumple con un modelo multiplicativo y se lo considera como confiable debido a que relaciona la tendencia, estacionalidad y el ruido, que son los factores de mayor influencia en una serie temporal, aplicando un modelo de extrapolación lineal se estudió la tendencia llegando a determinar mediante los datos históricos que la $T=$ tendecia es igual a $T=204,28+1.0956 * t$, sabiendo que $t=$ serie de tiempo

- Mediante medias móviles de grado tres se ha realizado el suavizado o ajuste o los parámetros de estacionalidad y ruido para que la estimación sea aceptada y los valores calculados se los considere dentro del margen de tolerancia, es por eso que se tiene en el modelo encontrado el índice de estacionalidad corregido IEC . 


\section{Referencias bibliográficas.}

Bocco, M. (2010). Funciones elementales para construir modelos matemáticos. 217.

Canabal, C., \& Marcel, A. (2009). Energía y cambio climático. Recuperado 4 de mayo de 2019, de Cerlalc website: https://cerlalc.org/rilvi/energia-y-cambio-climatico/

Cano, J. E. S. (2014). Gobernanza estratégica para el cambio de la matriz productiva y energética del caso del Ecuador. 20.

Cortés, E. A., \& Villamizar, G. O. (2000). Apuntes sobre energía y recursos energéticos. UNAB.

ECONOMÍA, C. D. (2015). FACULTAD DE CIENCIAS ECONÓMICAS. 84.

Garrido, A. A. (2009). La energía como elemento esencial de desarrollo. 61.

Gras, J. A. (2001). Diseños de series temporales: técnicas de análisis. Edicions Universitat Barcelona.

Henley, E. J. (1973). Cálculo de balances de materia y energía: métodos manuales y empleo de máquinas calculadoras. Reverte.

Lazo, L. (s.f.). MODELOS MATEMATICOS. Recuperado de https://www.academia.edu/7309281/MODELOS_MATEMATICOS

López, J. C. C. (2006). Problemas y modelos matemáticos para la administración y dirección de empresas. Editorial de la UPV.

Martínez, C. D. la F., Machín, M. C., Ruiz, J. L. G., Martínez, P. J., Rincón, T. O. D., Muñiz, T. J. R., ... López, M. J. G. (2012). Construcción de modelos matemáticos y resolución de problemas. Ministerio de Educación.

Navarro, F. S. (2011). El reto energético: Gestionando el legado de Prometeo. Universitat de València.

Peña, D. (2010). Análisis de series temporales. Alianza Editorial. 
Pérez, E. M., \& Ingeniero, D. (2007). ENERGÍAS RENOVABLES SOSTENIBILIDAD Y CREACIÓN DE EMPLEO. 35.

Prades, A. (1997). Energía, tecnología y sociedad. Ediciones de la Torre.

Recalde, M. Y. (2010). TESIS DE DOCTORADO EN ECONOMÍA. 252.

Roldán, J., \& Viloria, J. R. (2008). Fuentes de Energía. Editorial Paraninfo.

UNA SERIE DE TIEMPO TIENE LAS SIGUIENTES COMPONENTES: (s. f.). $\begin{array}{llllll}\text { Recuperado } & 4 & \text { de } & \text { mayo } & \text { de } & 2019,\end{array}$ http://matematicas.reduaz.mx/home/Docentes/ltrueba/Series/admon4.htm

Varsavsky, O., \& Calcagno, A. E. (1971). América Latina: modelos matemáticos; ensayos de aplicación de modelos de experimentación numérica a la política económica y las ciencias sociales. Editorial Universitaria.

Visitación, G. J. M., \& Andrés, C. S. P. (2014). DISEÑOS EXPERIMENTALES DE SERIES TEMPORALES. Editorial UNED. 


\section{PARA CITAR EL ARTÍCULO INDEXADO.}

Mazón Fierro, G., Calderón Limaico, P., Villa Uvidia, R., \& Villamarín Padilla, J. (2019). Modelo matemático para estimar la producción de la energía primaria en Ecuador. Ciencia Digital, 3(2.2), 118-131. https://doi.org/10.33262/cienciadigital.v3i2.2.464

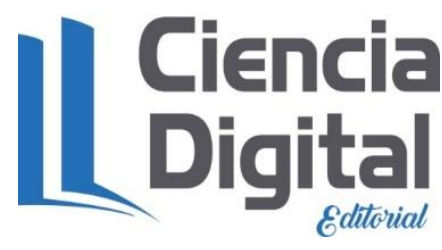

El artículo que se publica es de exclusiva responsabilidad de los autores y no necesariamente reflejan el pensamiento de la Revista Ciencia Digital.

El artículo queda en propiedad de la revista y, por tanto, su publicación parcial y/o total en otro medio tiene que ser autorizado por el director de la Revista Ciencia Digital.
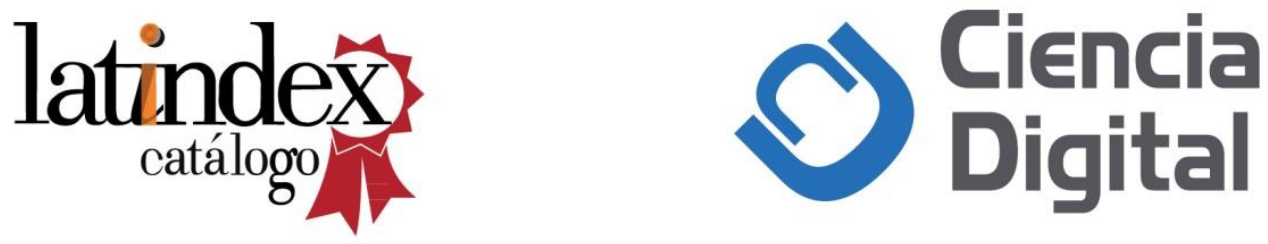